\title{
Reevaluating the Effectiveness of Corneal Collagen Cross- linking and Its True Biomechanical Effect in Human Eyes
}

\author{
Damien Gatinel
}

\section{ABSTRACT}

The induction of cross-links in corneal tissue appears to be a promising technique to increase its stiffness and this has been the basis of treatment of keratoconus $(\mathrm{KC})$ and corneal ectatic disease. However, there exists a striking discrepancy between the reported biomechanical effects of corneal collagen cross-linking (CXL) in vitro compared to in vivo, and this has not received much attention in the literature.

Despite the documentation of an increase in corneal stiffness in vitro by many investigators, reports that provide evidence of measurable and consistent biomechanical changes in corneal rigidity in vivo after $C X L$ are lacking. Indeed, the absence of documented in vivo biomechanical improvement in CXL-treated corneas is a conundrum, which needs to be further explored. To explain this discrepancy, it has been postulated that biomechanical changes induced by CXL are too subtle to be measured by currently available diagnostic tools or have characteristics not discernible by these technologies. However, the dynamic bidirectional applanation device (Ocular Response Analyzer) and dynamic Scheimpflug analyzer instruments (Corvis ST) have demonstrated the ability to quantify even subtle biomechanical differences in untreated KC corneas of different ectatic degree, and document the reduction in corneal hysteresis $(\mathrm{CH})$ and corneal resistance factor (CRF) in situations where the corneal stiffness is reduced, such as after laser in situ keratomileusis and surface ablation procedures. It has also been possible to demonstrate an altered $\mathrm{CH}$ and $\mathrm{CRF}$ in patients with diabetes, smoking habit, glaucoma, Fuchs' dystrophy, and corneal edema. It is puzzling that these diagnostic tools could document subtle biomechanical changes in these situations, yet fail to measure the purported changes induced by CXL on corneas with progressive KC. This failure to document significant and consistent biomechanical changes in corneal rigidity could suggest that CXL does not induce a simple reversal of the particular biomechanical deficits that characterize $\mathrm{KC}$, or make the cornea significantly more resistant to bending forces as has been widely postulated. The absence of measurable biomechanical change in living KC corneas after CXL could be a consequence of biomechanical strengthening which is insignificant compared to the marked weakening caused by preexisting alteration of the collagen structure, disorganization of collagen fiber intertwining, and compromised structural-mechanical homogeneity that are hallmarks of keratoconic disease, especially in corneas with progressive KC.

\footnotetext{
Head

Department of Anterior Segment and Refractive Surgery Rothschild Ophthalmic Foundation, Paris, France

Corresponding Author: Damien Gatinel, Head, Department of Anterior Segment and Refractive Surgery, Rothschild Ophthalmic, Foundation, CEROC, 25 Rue Manin, 75019 Paris France, Phone: +33148036482, e-mail: gatinel@gmail.com
}

The changes in the cornea induced by $C X L$ that have been described in vivo may instead be driven by a wound healing process in response to the removal of the corneal epithelial layer and subsequent exposure to riboflavin and ultraviolet-A (UVA). This paper will present evidence that sustains this hypothesis.

Keywords: Corneal biomechanics, Corneal epithelium, Crosslinking, Ectasia, Hysteresis, Keratoconus.

How to cite this article: Gatinel D. Reevaluating the Effectiveness of Corneal Collagen Cross-linking and Its True Biomechanical Effect in Human Eyes. Int J Kerat Ect Cor Dis 2017;6(1):34-41.

\section{Source of support: Nil}

Conflict of interest: None

\section{INTRODUCTION}

Spoerl et al showed that the biomechanical behavior of corneas obtained from enucleated porcine eyes could be altered by riboflavin and ultraviolet (UV) irradiation. ${ }^{1}$ In keratoconus (KC), where the corneal stiffness is reduced, the concept of increased stability of the cornea afforded by artificial cross-linking (with radiation or chemical agents) led to the possibility of a potential conservative treatment for KC. The induction of cross-links in corneal tissue to possibly increase its stiffness was further explored in animal studies. ${ }^{2}$ There was subsequently ample evidence in vitro demonstrating the alteration in biomechanical behavior of the cornea by combined riboflavin/ultraviolet-A (UVA) induced collagen cross-linking.

By the late 90s, subsequent studies showed that cross-linking with riboflavin and UV could potentially stabilize the human cornea. The initial results of the first clinical trials suggested that CXL could provide a useful conservative treatment modality to retard or arrest the progression of KC and post-LASIK keratectasia. ${ }^{3,4}$ In contrast to preliminary in vitro studies, the evaluation of biomechanical changes in vivo could not be performed in the first clinical studies, because instruments for measuring the corneal biomechanical properties in vivo did not exist then. Hence, the parameters used to monitor CXL efficacy and safety in early clinical studies were visual acuity testing, corneal topography and measurements of endothelial cell density, instead of the key parameter determining the efficacy of the strengthening effect of CXL on the cornea, which is biomechanics. 


\section{CLINICAL EVALUATION OF THE BIOMECHANICAL CHANGES OCCURRING IN VIVO AFTER CXL}

In vivo evaluation of the biomechanical properties of the cornea was made possible in 2005 with the introduction of the Ocular Response Analyzer (ORA; Reichert, Buffalo, New York, USA) in clinical practice. ${ }^{5}$ This instrument uses a rapid air pulse to indent the cornea and an electro-optical system to monitor the bidirectional deformation of the cornea. Two primary indicators of corneal viscoelastic behavior are extracted from this measurement process, namely corneal hysteresis $(\mathrm{CH})$ and corneal resistance factor (CRF). The method of operation of the device and evidence for the clinical utility of these parameters are described extensively elsewhere. ${ }^{5}$ Keratoconus, Fuchs' dystrophy, Glaucoma, Marfan syndrome, and post-LASIK patients have been found to have low $\mathrm{CH}$ and $\mathrm{CRF}^{6-13}$

The corneal visualization Scheimpflug technology tonometer (Corvis ST tonometry: CST; Oculus, Wetzlar, Germany) was introduced more recently and allows quantitative and visual assessment of the biomechanical properties of the cornea. ${ }^{14}$ The Corvis ST is a noncontact tonometer with an integrated ultra-high-speed Scheimpflug camera, enabling the direct visualization of corneal movement during the application of a rapid air-puff. The instrument's high speed camera is capable of taking two-dimensional images of the cross-section of the cornea during its deformation. The device reports numerous parameters, such as amplitude of corneal deformation, area of applanation, and deformation velocity, which provide information on corneal biomechanical properties.

Both ORA and CST have been employed to assess the effects of corneal CXL on KC.

A pilot study was conducted to investigate the biomechanical changes after CXL with riboflavin/UV-A using the ORA. There were no significant differences in corneal biomechanical properties as measured with the parameters $\mathrm{CH}$ and $\mathrm{CRF} .{ }^{15}$ In another study, Sedaghat et $\mathrm{al}^{16}$ compared $\mathrm{CH}$ and CRF before and after CXL for $\mathrm{KC}$ and concluded that although previous in vitro studies found a change in corneal rigidity, their study found no significant change in $\mathrm{CH}$ or $\mathrm{CHF}$ measured by biomechanical waveform analysis.

These results were echoed in a study by Gkika et al, in which no significant change in the CRF parameters were found in keratoconic eyes before and after riboflavin/ UVA corneal CXL. ${ }^{17}$

No significant changes in $\mathrm{CH}$ and $\mathrm{CRF}$ were found after a 24 months follow-up, in another report where 57 eyes of 55 patients with progressive KC were consecutively treated with UVA- riboflavin CXL. The eyes were also examined with the corneal topographer Penta$\mathrm{cam}^{18}$ and surprisingly, in the subgroup of patients with decreased Kmax readings 24 months after treatment, both $\mathrm{CH}$ and CRF showed a significant reduction. Spoerl et al could not find significant changes in $\mathrm{CH}$ and CRF after cross-linking, and concluded that keratoconic corneas display altered biomechanical properties, which remain different from those observed in healthy corneas. ${ }^{19}$ Analyzing the waveform signs of the ORA measurements, they found that the area under peak 2 had significantly increased after CXL, suggesting that this parameter could more sensitively detect biomechanical changes after CXL than $\mathrm{CH}$ or CRF alone. Kiliç and Roberts have reported a significant increase in the height of peak 1 after transepithelial cross-linking, and attributed it to an increase in corneal stiffness. ${ }^{20}$ These findings have not been replicated since. The increase in the height of peak 1 or the area under peak 2 could be the result of a modified corneal surface which provided better reflectivity from improved corneal homogeneity and regularity, which has been reported after CXL. ${ }^{21}$

A prospective study was conducted to evaluate the correlation between corneal biomechanical and morphological data in healthy eyes, eyes that underwent myopic photorefractive keratectomy (PRK), eyes with $\mathrm{KC}$, and keratoconic eyes that underwent corneal CXL. ${ }^{22}$ Tomographic (Pentacam, Oculus, Wetzlar, Germany) and biomechanical (Corvis ST, Oculus, Wetzlar, Germany) evaluations were performed. Corneas that were affected by KC and keratoconic eyes that underwent CXL seemed to be easier to applanate, compared to healthy and post PRK eyes, showing a lower resistance to deformation. Surprisingly, the resistance to deformation was even less for eyes post-CXL than for untreated KC corneas. The post-CXL corneas also took more time to return to the applanation position and recover its original shape. Interestingly, corneas after PRK showed similar resistance to deformation as normal eyes, suggesting that the corneal thinning induced by PRK did not alter the biomechanical properties of the cornea much from that of healthy eyes.

Bak-Nielsen et al also described corneal deformation properties using rapidly applied forces via an air jet with ultra-high speed Scheimpflug technology in keratoconic eyes ${ }^{23}$ Patients with both untreated and CXL-treated KC were significantly different from normal patients with respect to certain deformation parameters, but again, no significant differences were found between patients with untreated KC and CXL-treated KC.

Tomita et al investigated shorter duration of UV light exposure in corneal CXL, based on the assumption that higher power delivered over shorter time periods could provide the same corneal strengthening as lower power 
over longer time periods. ${ }^{24}$ Regardless of the surgical protocol, no statistically significant difference between several biomechanical parameters could be recorded before and after CXL by the ORA and Corvis ST. There was also no significant difference in the measured parameters between the accelerated and conventional corneal CXL.

The results of these studies strongly suggest that with the current available technology in clinical practice, no significant and reproducible change in clinical parameters related to corneal biomechanics can be demonstrated in eyes that have undergone CXL for progressive KC.

\section{HOW THEN CAN WE EXPLAIN THE LACK OF CLEARLY DOCUMENTED BIOMECHANICAL CHANGES AFTER CXL?}

To account for these surprising results, several hypotheses may be invoked.

First, it has been postulated that biomechanical changes induced by CXL are too subtle to be measured by the ORA and Corvis ST, or have attributes not wellcharacterized by these technologies. However, it has been demonstrated that the ORA and Corvis ST have the capacity to record even subtle biomechanical differences in nontreated keratoconic corneas of different ectatic degree and in other various clinical situations. ${ }^{6-8}$ The $\mathrm{CH}$ and $\mathrm{CRF}$ have been shown to be altered in patients with diabetes (correlated with HbA1c levels), ${ }^{25}$ in smokers, ${ }^{26,27}$ Marfan syndrome, ${ }^{12}$ and in other instances where altered corneal biomechanics consistent with modified collagen properties would be expected. ${ }^{28}$ The observation of the reduction in $\mathrm{CH}$ and $\mathrm{CRF}$ values after LASIK and surface ablation procedures have also been demonstrated. ${ }^{29-32}$ The same authors who concluded that the Corvis ST may not be reliable to quantify the effect of CXL in KC eyes have published another study where they showed that it was possible to evaluate the corneal biomechanical properties after LASIK, ReLEx flex, and ReLEx smile. ${ }^{33}$

If the changes induced by CXL on parameters, such as $\mathrm{CH}$ and $\mathrm{CRF}$ are too subtle to be documented unlike those in the abovementioned clinical situations, then would it not be logical to conclude that the effect of CXL on corneal biomechanics may be too modest to be clinically relevant?

An alternative argument would be that the $\mathrm{CH}$ and $\mathrm{CRF}$ are inadequate metrics to demonstrate the possible stiffening achieved by CXL on keratoconic corneas. These parameters are not only influenced by the viscoelastic properties of the corneal tissue, but other parameters, such as corneal thickness and intraocular pressure. The cornea is a highly complex anisotropic tissue with a distinctive collagen fiber arrangement interacting with a complex collagen matrix. During ORA and Corvis ST measurements, the force acts perpendicular to the cornea, and the obtained signals are determined by its bending stiffness. The resistance to the bending of the cornea depends on the collagen fibers and the ground substance in which the fibers are embedded, which consists of glycosaminoglycans and proteoglycans. If the viscosity of the cornea is determined mainly by the ground substance, the creation of links between collagen fibrils or fibers may not significantly modify the value of $\mathrm{CH}$ and CRF parameters. The lower $\mathrm{CH}$ values observed after CXL may in fact be derived from decreased glycosaminoglycans due to cell death.

The absence of measurable biomechanical changes in living KC corneas after CXL contrasts with the results of ex vivo experimentations, which show significant stiffening effects with standard and some modified CXL protocols, including evidence of increased elastic modulus and increased stiffness. It is possible that in vivo human corneas with progressive $\mathrm{KC}$ do not respond to $\mathrm{CXL}$ in the same manner as in animal models. This discrepancy could be due to CXL inducing insignificant mechanical strengthening compared to the marked weakening caused by the preexisting alteration of the collagen structure in progressive $\mathrm{KC}$. The disorganization of collagen fiber intertwining and the compromised structuralmechanical homogeneity induced by the $\mathrm{KC}$ disease may be too overwhelming in progressive $\mathrm{KC}$ corneas to be improved by CXL in any of its current (i.e., accelerated or conventional) in vivo modalities. In experimental and theoretical models, the biomechanical behavior of corneal structures is estimated through stretch forces parallel to the corneal surface direction. Because of the lack of a comprehensive and cohesive theoretical model, bending forces are not considered.

In $\mathrm{KC}$, the impact of intensive eye rubbing may be the predominant, if not necessary, deformation mechanism responsible for the resultant corneal ectasia. ${ }^{34}$ During eye rubbing episodes, the intraocular pressure is markedly elevated, and the cornea is squeezed between compressive forces, resulting in significant corneal tissue trauma. The shearing forces imposed by the fingers or knuckles (rotary or grinding movements on the corneal structure) may alter the fiber adherence and reduce the viscosity of the ground substance of the corneal matrix. In the central corneal region, which is directly exposed to the rubbing trauma, the resistance to bending may be reduced focally, causing local arching of the cornea and the characteristic deformation observed in KC corneas (steep paracentral area surrounded by a flatter zone). The CXL may improve the resistance of the cornea to lateral forces, but not bending forces. Indirect evidence of the predominant 
effect of bending compared to lateral shearing forces in $\mathrm{KC}$ deformation is provided by a study, which showed that the surface area of $\mathrm{KC}$ corneas is remarkably insensitive to curvature change near the vertex. ${ }^{35}$ Flattening is seen in the periphery of corneas with $\mathrm{KC}$ suggests that biomechanical coupling compensates for any increase in curvature occurring in the region of the cone itself. This study also suggested that $\mathrm{KC}$ is not a true ectasia unlike keratoglobus, but is instead a specialized type of warpage, at least in the mild to moderate forms of the disease.

Shear wave elastography is a promising technique for rapid evaluation of corneal stiffness. This method has been used ex vivo to measure the corneal biomechanics of animal corneas. However, no data regarding possible in vivo stiffening after CXL on KC corneas has been evidenced $_{\text {yet. }}^{36,37}$

\section{THE NEED TO REEVALUATE THE EFFECTIVENESS OF CXL: AN ALTERNATIVE HYPOTHESIS}

With the current evidence, it may be challenging for clinicians and scientists to accept the purported biomechanical effects induced by CXL. In KC, post-corneal refractive surgery and corneal edema where corneal stiffness is reduced, ${ }^{6-9,13,29-32} \mathrm{CH}$ and CRF are concomitantly reduced. Why then do $\mathrm{CH}$ and $\mathrm{CRF}$ not be increased in CXL-treated corneas if the primary effect of CXL is to stiffen the cornea? It is difficult to comprehend that the stiffening caused by CXL in keratoconic corneas may have such peculiar characteristics that these changes are undetectable by clinical instruments otherwise proven effective for detecting subtle alterations in corneal viscoelastic properties in KC and other instances with altered biomechanics.

In classical mechanics, the dynamical laws are deterministic and reversible with time. If the reduction in $\mathrm{CH}$ that occurs during the evolution of $\mathrm{KC}$ or after corneal refractive surgery can be monitored by an instrument, this same instrument should be able to detect the increase in $\mathrm{CH}$ following the reduction of $\mathrm{KC}$ severity or "reversal" of the refractive procedure. This logically suggests that if CXL could significantly impact KC corneas by making significant biomechanical alterations to counter the weakening in progressive disease, instruments such as the ORA or the Corvis ST should be able to identify these biomechanical changes, just as they are able to when the biomechanics are degraded by KC progression, corneal lamellar surgery, or surface refractive surgical procedures.

As the intended purpose of CXL is to increase the rigidity of the treated cornea by creating chemical bonds between collagen fibers, the lack of documented biomechanical improvement in $\mathrm{CH}$ and $\mathrm{CRF}$ parameters could be regarded as lack of effectiveness. ${ }^{38}$ Before sound evidence of increased corneal viscoelastic properties is presented via in vivo quantifiable metrics, one cannot exclude the simple yet provocative hypothesis that CXL fails to significantly alter the biomechanical properties of keratoconic corneas.

\section{CAUTION IN INTERPRETING THE TOPOGRAPHIC RESPONSE TO CXL}

Parameters such as corneal curvature, visual acuity, and topographic changes after CXL have been shown to be influenced by CXL. These changes were initially not directly intended by the CXL protocols, which were aimed primarily at increasing corneal stiffness. In many studies, the outcomes of CXL were evaluated with non-biomechanical investigational tools only, such as keratometry, topographic astigmatism magnitude, etc. In contrast to the lack of biomechanical alterations, significant topographical changes have been reported after CXL in patients with KC and corneal ectasia. ${ }^{18,39-42}$ There were significant improvements in the index of surface variance, index of vertical asymmetry, KC index, and minimum radius of curvature at 1 year. ${ }^{43} \mathrm{~A}$ reduction of the corneal and ocular high order aberrations was also observed after CXL, suggesting an improvement in corneal shape. ${ }^{44}$

The amplitude and the delayed onset response of topographical improvements strongly suggests that the improvements in keratometric readings and visual quality reported after CXL may be due to healing mechanisms as described below.

The threshold values of keratometric changes used to determine KC progression or post-CXL improvement should also be carefully defined. Caution should be taken when interpreting the topographies of such patients because of the higher variability of topographic measurements in KC patients. We have recently assessed the repeatability of the corneal topography functions of Orbscan IIz (Bausch and Lomb, Rochester, New York), OPD-Scan III (Nidek, Gamagori, Japan), and iTrace (Tracey Technologies, Houston, TX) in keratoconic eyes and in a control group of normal patients. ${ }^{45}$ For the maximum keratometry measurement, repeatability limit was $1.73,1.49$, and 1.41D in the stage I-IV keratoconic eyes group, 1.11, 1.02, and $0.98 \mathrm{D}$ in the stage I-II keratoconic eyes group, and $0.61,0.37$, and $1.02 \mathrm{D}$ in the normal eyes group with Orbscan II, OPD-Scan III, and iTrace respectively. These results illustrate the fact that topographies performed in keratoconic eyes are less repeatable than those performed in normal eyes. The higher variability should be considered before performing any treatment, 
as well as when evaluating its effectiveness. A recent meta-analysis aiming to assess the efficacy and safety of epithelial removal (ER) and transepithelial corneal CXL for the treatment of $\mathrm{KC}$ reported that based on the analysis of 27 studies on ER CXL, the median value of the reduction in maximal keratometry was-1.01D $(-0.14$ to $-6.16 \mathrm{D}){ }^{46}$ The conclusions in many of these studies aimed at evaluating preoperative progression and postCXL stability may be invalidated if the magnitude of the variations of the maximum keratometry in $\mathrm{KC}$ eyes failed to exceed the repeatability limits of the devices used.

\section{COULD EPITHELIAL WOUND HEALING ACCOUNT FOR MOST OF THE CHANGES OBSERVED AFTER CXL?}

Cross-linking of collagen refers to the ability of collagen fibrils to form chemical bonds with adjacent fibrils. This requires both UV light and a photosensitizer to strengthen chemical bonds in the cornea; the simultaneous presence of both riboflavin and UVA is required to produce significant cross-linking of the fibrils. Hence, suppression of UV irradiation will result in interruption of the cross-linking reaction. The purported biomechanical and topographic changes incurred by CXL should, therefore, happen during the procedure itself, and be measurable within a few days, right after the re-epithelialization period. Surprisingly, the initial topography changes are usually marked by a mild central steepening, whereas the onset of mild flattening occurs typically months after the CXL procedure. ${ }^{18,41,42}$ This late onset flattening that has been attributed to the primary effect of CXL should instead be attributed to a healing response, as any chemically induced response from the concomitant UVA irradiation (as in CXL) would have occurred during the procedure itself, or shortly after.

A similar central flattening with significant gain in corrected distance visual acuity occurs frequently after phototherapeutic keratectomy, even when shallow ablation with no refractive correction is performed, as for recurrent erosion syndrome. ${ }^{47}$ Based on Munnerlyn's simplified equation thickness/ablation depth, $(\mu \mathrm{m})=1 / 3$ $\times$ intended correction $\left.(\mathrm{D}) \times[\mathrm{OZ} \text { diameter }(\mathrm{mm})]^{2}\right\}$ a reduction of 2 diopters (D) within the central 3-mm zone could result from a variation of less than $10 \mu \mathrm{m}$ in epithelial thickness within this optical zone diameter. Interestingly, the prevalent role of epithelium regrowth in the changes seen post-CXL is substantiated by the fact that the effects of transepithelial (epi on) CXL appear to be less pronounced than the effects of CXL with de-epithelialization (epi off) as described in the literature. ${ }^{46,48-50}$ It should be reasonable to conclude, therefore, that the variation in keratometric readings and visual quality observed after
CXL may be non-biomechanical and instead be related to epithelial remodeling.

We have conducted a study to further investigate the role of the corneal epithelium on the topography of the anterior surface of the cornea. ${ }^{51}$ In this study, preoperative OPD-scan topography was performed on each eye 10 minutes before PRK (for myopia) and the administration of topic anesthesia. Following removal of the epithelium, the obtained specular image of the denuded corneal surface allowed the computation of the topography of the Bowman's membrane in normal myopic eyes. In 90 low to moderately myopic eyes of 51 patients, we found that the topography of Bowman's layer was significantly steeper than that at the epithelial surface. The epithelial layer tended to decrease slightly the magnitude of astigmatism and prolateness of Bowman's layer. In eyes with $\mathrm{KC}$, this compensatory effect of the corneal epithelium was found to be more pronounced. ${ }^{52}$ Interestingly, in these diseased corneas, the magnitude of the reported changes in keratometry after de-epithelialization were higher than that reported after cross-linking. In these keratoconic eyes, the central epithelium was never totally removed before the advent of the CXL procedure, and yet, could remodel itself to provide the anterior corneal surface with an improved contour. This strongly suggests that regrowth of corneal epithelium may account for the slight topographic changes that have been reported in the long postoperative course after CXL.

\section{WHAT IS THE PLACE OF CXL TODAY AND IN THE FUTURE?}

Since its introduction to ophthalmic practice in $2003,{ }^{3}$ a number of reports have concluded that CXL may slow or halt the progression of KC and post-LASIK ectasia. In contrast, the Cochrane Database of Systematic Reviews conclusion was less convincing and stated that the evidence for the use of CXL in the management of KC was limited due to the lack of properly conducted randomized controlled trials. ${ }^{53}$ However, these considerations may still be insufficient to discourage ophthalmologists performing CXL in patients with progressive KC. A recent editorial in the journal Ophthalmology concluded that the recent United States Food and Drug Administraton approval for the technique might have been triggered more by an unmet medical need rather than evidence based medicine. ${ }^{54}$ Currently, other than controlling risk factors for progression (i.e., eye rubbing), there are no other alternative conservative solutions to halt the progression of $\mathrm{KC}$.

A secondary benefit of CXL could be that having the CXL procedure may induce a reluctance for the patient to touch or rub his or her eyes, and this could help to stabilize or halt progression of the keratoconic disease. 


\section{CONCLUSION}

Regardless of the place of CXL in KC management, the inability to document alleged biomechanical improvement after CXL with currently available methods in KC patients should invite skepticism from clinicians and researchers alike. In science, we are advised to take the null hypothesis as the default position. In the context of cross-linking studies, the null hypothesis would essentially be that CXL has no biomechanical effect on the cornea. Analysis of current literature provides no strong evidence against the null hypothesis, therefore, only a weak conclusion can be made until proven otherwise that the CXL is unable to strengthen the cornea and has failed to achieve the goal it has been conceived for.

Despite the apparent lack of evidence of clinically relevant corneal biomechanical changes, the widespread positive perception of CXL among the ophthalmic community may reflect a confirmation bias, which is the tendency to search for or interpret information in a way that confirms one's preconceptions.

With the current protocols, the evidence shows that only corneal curvature, visual acuity, and topography can be influenced by CXL. These parameters, and not biomechanical strengthening, have been used by both surgeons and patients as yardsticks to qualify success of CXL and justify the need to perform the procedure. Ignoring the evidence that does not support the preconception that CXL is a biomechanically efficient strengthening technique in vivo may preclude researchers to improve and refine current treatment modalities. In fact, the author believes the mysterious discrepancy between the in vivo and in vitro biomechanical changes of the cornea after CXL warrants further attention and investigation. The documented effects of epithelial wound healing after CXL should be explored in depth as it may explain most if not all the topographic changes observed over time. In this field, the newer high resolution OCT techniques may be of particular interest. More importantly, elucidating why the current CXL protocols have failed to reverse the clinically measurable corneal biomechanical impairment in KC eyes may be a prerequisite to understanding and discovering new, truly efficacious, and biomechanically sound CXL techniques for ectatic corneas.

\section{ACKNOWLEDGMENT}

Author would like to thank Cordelia Chan, MD, for assisting and providing critical revision of the manuscript.

\section{REFERENCES}

1. Spoerl E, Huhle M, Seiler T. Induction of cross-links in corneal tissue. Exp Eye Res 1998 Jan;66(1):97-103.
2. Spoerl E, Seiler T. Techniques for stiffening the cornea. J Refract Surg 1999 Nov-Dec;15(6):711-713.

3. Wollensak G, Spoerl E, Seiler T. Riboflavin/ultraviolet-ainduced collagen crosslinking for the treatment of keratoconus. Am J Ophthalmol 2003 May;135(5):620-627.

4. Kohlhaas M, Spoerl E, Speck A, Schilde T, Sandner D, Pillunat LE. [A new treatment of keratectasia after LASIK by using collagen with riboflavin/UVA light cross-linking]. Klin Monbl Augenheilkd 2005 May;222(5):430-436.

5. Luce DA. Determining in vivo biomechanical properties of the cornea with an ocular response analyzer. J Cataract Refract Surg 2005 Jan;31(1):156-162.

6. Shah S, Laiquzzaman M, Bhojwani R, Mantry S, Cunliffe I. Assessment of the biomechanical properties of the cornea with the ocular response analyzer in normal and keratoconic eyes. Invest Ophthalmol Vis Sci 2007 Jul;48(7):3026-3031.

7. Saad A, Lteif Y, Azan E, Gatinel D. Biomechanical properties of keratoconus suspect eyes. Invest Ophthalmol Vis Sci 2010 Jun;51(6):2912-2916.

8. Ali NQ, Patel DV, McGhee CN. Biomechanical responses of healthy and keratoconic corneas measured using a noncontact Scheimpflug-based tonometer.Invest Ophthalmol Vis Sci 2014 May 15;55(6):3651-3659.

9. del Buey MA, Cristóbal JA, Ascaso FJ, Lavilla L, Lanchares E. Biomechanical properties of the cornea in Fuch's' corneal dystrophy. Invest Ophthalmol Vis Sci 2009 Jul;50(7): 3199-3202.

10. Prata TS, Lima VC, De Moraes CG, Guedes LM, Magalhães FP, Teixeira SH, Ritch R, Paranhos A Jr. Factors associated with topographic changes of the optic nerve head induced by acute intraocular pressure reduction in glaucoma patients. Eye (Lond) 2011 Feb;25(2):201-207.

11. Deol M, Taylor DA, Radcliffe NM. Corneal hysteresis and its relevance to glaucoma. Curr Opin Ophthalmol 2015 Mar;26(2):96-102.

12. Beene LC, Traboulsi EI, Seven I, Ford MR, Sinha Roy A, Butler RS, Dupps WJ Jr. Corneal deformation response and ocular geometry: a noninvasive diagnostic strategy in Marfan syndrome. Am J Ophthalmol 2016 Jan;161:56-64.e1.

13. Chen S, Chen D, Wang J, Lu F, Wang Q, Qu J. Changes in ocular response analyzer parameters after LASIK. J Refract Surg 2010 Apr;26(4):279-288.

14. Koprowski R. Automatic method of analysis and measurement of additional parameters of corneal deformation in the Corvis tonometer. Biomed Eng Online 2014 Nov;13:150.

15. Goldich Y, Barkana Y, Morad Y, Hartstein M, Avni I, Zadok D. Can we measure corneal biomechanical changes after collagen cross-linking in eyes with keratoconus?--a pilot study. Cornea 2009 Jun;28(5):498-502.

16. Sedaghat M, Naderi M, Zarei-Ghanavati M. Biomechanical parameters of the cornea after collagen crosslinking measured by waveform analysis. J Cataract Refract Surg 2010 Oct;36(10):1728-1731.

17. Gkika MG, Labiris G, Kozobolis VP. Tonometry in keratoconic eyes before and after riboflavin/UVA corneal collagen crosslinking using three different tonometers. Eur J Ophthalmol 2012 Mar-Apr;22(2):142-152.

18. De Bernardo M, Capasso L, Lanza M, Tortori A, Iaccarino S, Cennamo M, Borrelli M, Rosa N. Long-term results of corneal collagen crosslinking for progressive keratoconus. J Optom 2015 Jul-Sep;8(3):180-186. 
19. Spoerl E, Terai N, Scholz F, Raiskup F, Pillunat LE. Detection of biomechanical changes, after corneal cross-linking using ocular response analyzer software. J Refract Surg 2011 Jun;27(6):452-457.

20. Kiliç A, Roberts C. Biomechanical and refractive results of transepithelial cross linking treatment in keratoconic eyes. Int J Kerat Ect Cor Dis 2012 May-Aug;1(2):53-56.

21. Vinciguerra $P$, Albè E, Trazza $S$, Rosetta $P$, Vinciguerra $R$, Seiler T, Epstein D. Refractive, topographic, tomographic, and aberrometric analysis of keratoconic eyes undergoing corneal crosslinking. Ophthalmology 2009 Mar;116(3): 369-378.

22. Lanza M, Cennamo M, Iaccarino S, Irregolare C, Rechichi M, Bifani M, Gironi Carnevale UA. Evaluation of corneal deformation analyzed with Scheimpflug based device in healthy eyes and diseased ones. Biomed Res Int 2014;2014:748671.

23. Bak-Nielsen S, Pedersen IB, Ivarsen A, Hjortdal J. Dynamic Scheimpflug-based assessment of keratoconus and the effects of corneal cross-linking. J Refract Surg 2014 Jun;30(6): 408-414.

24. Tomita M, Mita M, Huseynova T. Accelerated versus conventional corneal collagen crosslinking. J Cataract Refract Surg 2014 Jun;40(6):1013-1020.

25. Scheler A, Spoerl E, Boehm AG. Effect of diabetes mellitus on corneal biomechanics and measurement of intraocular pressure. Acta Ophthalmol 2012 Sep;90(6):e447-451.

26. Schweitzer C, Korobelnik JF, Boniol M, Cougnard-Gregoire A, Le Goff M, Malet F, Rougier MB, Delyfer MN, Dartigues JF, Delcourt C. Associations of biomechanical properties of the cornea with environmental and metabolic factors in an elderly population: the ALIENOR study. Invest Ophthalmol Vis Sci 2016 Apr1;57(4):2003-2011.

27. Kilavuzoglu AE, Celebi AR, Altiparmak UE, Cosar CB. The effect of smoking on corneal biomechanics. Curr Eye Res 2017 Jan;42(1):16-20.

28. Spörl E, Terai N, Haustein M, Böhm AG, Raiskup-Wolf F, Pillunat LE. [Biomechanical condition of the cornea as a new indicator for pathological and structural changes]. Ophthalmologe 2009 Jun;106(6):512-520.

29. Qazi MA, Sanderson JP, Mahmoud AM, Yoon EY, Roberts CJ, Pepose JS. Postoperative changes in intraocular pressure and corneal biomechanical metrics Laser in situ keratomileusis versus laser-assisted subepithelial keratectomy. J Cataract Refract Surg 2009 Oct;35(10):1774-1788.

30. Kamiya K, Shimizu K, Ohmoto F. Comparison of the changes in corneal biomechanical properties after photorefractive keratectomy and laser in situ keratomileusis. Cornea 2009 Aug;28(7):765-769.

31. Kamiya K, Shimizu K, Ohmoto FTime course of corneal biomechanical parameters after laser in situ keratomileusis. Ophthalmic Res 2009;42(3):167-171.

32. Barbara R, Nassar A, Zadok D, Barbara A. Corneal biomechanical properties post-LASEK for the correction of myopia. Int JKerat Ect Cor Dis2014 Jan-Apr;3(1):23-28.

33. Pedersen IB, Bak-Nielsen $S$, Vestergaard AH, Ivarsen A, Hjortdal J. Corneal biomechanical properties after LASIK, ReLEx flex, and ReLEx smile by Scheimpflug-based dynamic tonometry. Graefes Arch Clin Exp Ophthalmol 2014 Aug;252(8):1329-1335.

34. Gatinel D. Eye rubbing, a sine qua non for keratoconus? Int J Kerat Ect Cor Dis 2016 Jan-Apr;5(1):6-12.
35. Smolek MK1, Klyce SD. Is keratoconus a true ectasia? An evaluation of corneal surface area. Arch Ophthalmol. 2000;118(9): 1179-1186.

36. Nguyen TM, Aubry JF, Fink M, Bercoff J, Tanter M. In vivo evidence of porcine cornea anisotropy using supersonic shear wave imaging. Invest Ophthalmol Vis Sci 2014 Oct.28;55(11):7545-7552.

37. Touboul D, Gennisson JL, Nguyen TM, Robinet A, Roberts CJ, Tanter M, Grenier N. Supersonic shear wave elastography for the in vivo evaluation of transepithelial corneal collagen cross-linking. Invest Ophthalmol Vis Sci 2014 Mar28;55(3): 1976-1984.

38. Gatinel D. Effectiveness of corneal collagen crosslinking in vivo for corneal stiffening. J Cataract Refract Surg 2014 Nov;40(11):1943-1944.

39. Goldich Y, Marcovich AL, Barkana Y, Mandel Y, Hirsh A, Morad Y, Avni I, Zadok D. Clinical and corneal biomechanical changes after collagen cross-linking with riboflavin and UV irradiation in patients with progressive keratoconus: results after 2 years of follow-up. Cornea 2012 Jun;31(6):609-614.

40. Raiskup-Wolf F, Hoyer A, Spoerl E, Pillunat LE. Collagen crosslinking with riboflavin and ultraviolet-A light in keratoconus: long-term results. J Cataract Refract Surg 2008 May;34(5):796-801.

41. Vinciguerra P, Albè E, Frueh BE, Trazza S, Epstein D. Twoyear corneal cross-linking results in patients younger than 18 years with documented progressive keratoconus. Am J Ophthalmol 2012 Sep;154(3):520-526.

42. Caporossi A, Mazzotta C, Baiocchi S, Caporossi T. Long-term results of riboflavin ultraviolet a corneal collagen crosslinking for keratoconus in Italy: the Siena eye cross study. Am J Ophthalmol 2010 Apr;149(4):585-593.

43. Greenstein SA, Fry KL, Hersh PS. Corneal topography indices after corneal collagen crosslinking for keratoconus and corneal ectasia: one-year results. J Cataract Refract Surg. 2011;37(7):1282-1290.

44. Greenstein SA, Fry KL, Hersh MJ, Hersh PS. Higher-order aberrations after corneal collagen crosslinking for keratoconus and corneal ectasia. J Cataract Refract Surg 2012 Feb;38(2):292-302.

45. Guilbert E, Saad A, Elluard M, Grise-Dulac A, Rouger H, Gatinel D. Repeatability of keratometry measurements obtained with three topographers in keratoconic and normal corneas. J Refract Surg 2016 Mar;32(3):187-192.

46. Shalchi Z, Wang X, Nanavaty MA. Safety and efficacy of epithelium removal and transepithelial corneal collagen crosslinking for keratoconus. Eye (Lond). 2015 Jan;29(1):15-29.

47. Starr M, Donnenfeld E, Newton M, Tostanoski J, Muller J, Odrich M. Excimer laser phototherapeutic keratectomy. Cornea 1996 Nov;15(6):557-565.

48. Vinciguerra P, Romano V, Rosetta P, Legrottaglie EF, Piscopo R, Fabiani C, Azzolini C, Vinciguerra R. Transepithelial iontophoresis versus standard corneal collagen crosslinking: 1-year results of a prospective clinical study. J Refract Surg 2016 Oct 1;32(10):672-678.

49. Stojanovic A, Chen X, Jin N, Zhang T, Stojanovic F, Raeder S, Utheim TP Safety and efficacy of epithelium-on corneal collagen cross-linking using a multifactorial approach to achieve proper stromal riboflavin saturation. J Ophthalmol 2012;2012:498435.

50. Filippello M, Stagni E, O'Brart D. Transepithelial corneal collagen crosslinking: bilateral study. J Cataract Refract Surg 2012 Feb;38(2):283-291. 
51. Salah-Mabed I, Saad A, Gatinel D. Topography of the corneal epithelium and Bowman layer in low to moderately myopic eyes. J Cataract Refract Surg 2016 Aug;42(8): 1190-1197.

52. Touboul D, Trichet E, Binder PS, Praud D, Seguy C, Colin J. Comparison of front-surface corneal topography and Bowman membrane specular topography in keratoconus. J Cataract Refract Surg 2012 Jun ;38(6):1043-1049.
53. Sykakis E, Karim R, Evans JR, Bunce C, Amissah-Arthur KN, Patwary S, McDonnell PJ, Hamada S. Corneal collagen crosslinking for treating keratoconus. Cochrane Database Syst Rev 2015 Mar24;(3) :CD010621.

54. Jeng BH, Farid M, Patel SV, Schwab IR. Corneal cross-linking for keratoconus: a look at the data, the food and drug administration, and the future. Ophthalmology 2016 Nov; 123(11):2270-2272. 